\title{
Isolated Singularities of Polyharmonic Operator in Even Dimension
}

\author{
Dhanya Rajendran ${ }^{\mathrm{a}}$, Abhishek Sarkar ${ }^{\mathrm{b}, *}$ \\ ${ }^{a}$ Department of Mathematics \\ Indian Institute of Science, Bangalore-560012, Karnataka, India. \\ ${ }^{b}$ TIFR Centre For Applicable Mathematics \\ Post Bag No. 6503, Sharda Nagar, Bangalore-560065, Karnataka, India.
}

\begin{abstract}
We consider the equation $\Delta^{2} u=g(x, u) \geq 0$ in the sense of distribution in $\Omega^{\prime}=\Omega \backslash\{0\}$ where $u$ and $-\Delta u \geq 0$. Then it is known that $u$ solves $\Delta^{2} u=g(x, u)+\alpha \delta_{0}-\beta \Delta \delta_{0}$, for some nonnegative constants $\alpha$ and $\beta$. In this paper we study the existence of singular solutions to $\Delta^{2} u=$ $a(x) f(u)+\alpha \delta_{0}-\beta \Delta \delta_{0}$ in a domain $\Omega \subset \mathbb{R}^{4}, a$ is a non-negative measurable function in some Lebesgue space. If $\Delta^{2} u=a(x) f(u)$ in $\Omega^{\prime}$, then we find the growth of the nonlinearity $f$ that determines $\alpha$ and $\beta$ to be 0 . In case when $\alpha=\beta=0$, we will establish regularity results when $f(t) \leq C e^{\gamma t}$, for some $C, \gamma>0$. This paper extends the work of Soranzo (1997) where the author finds the barrier function in higher dimensions $(N \geq 5)$ with a specific weight function $a(x)=|x|^{\sigma}$. Later we discuss its analogous generalization for the polyharmonic operator.
\end{abstract}

Keywords: Elliptic system; polyharmonic operator; existence of solutions; singularity 2010 MSC: 35J40, 35J61, 35J91

\section{Introduction}

Isolated singularities of elliptic operators are studied extensively, see for eg. [2],[10], [14], [15] and [16]. In this paper we wish to address the following problem and the questions related to it for the biharmonic(polyharmonic) operator in $\mathbb{R}^{4}\left(\mathbb{R}^{2 m}\right)$ :-

Question: If a non negative measurable function $u$ is known to solve a PDE in the sense of distribution in a punctured domain, then what can one say about the differential equation satisfied by $u$ in the entire domain?

In [2], Brezis and Lions answered this question for the Laplace operator with the assumption that

$$
0 \leq-\Delta u=f(u) \text { in } \Omega \backslash\{0\}, u \geq 0, \liminf _{t \rightarrow \infty} \frac{f(t)}{t}>-\infty, \Omega \subset \mathbb{R}^{N} .
$$

With the above hypotheses it was proved that both $u$ and $f(u)$ belong to $L^{1}(\Omega)$, and satisfy $-\Delta u=f(u)+\alpha \delta_{0}$, for some $\alpha \geq 0$. For the dimension $N \geq 3$, P.L.Lions[10] found a sharp condition on $f$ that determines whether $\alpha$ is zero or not in the previous expression. In [5], the authors further extended the result for dimension $N=2$ by finding the minimal growth rate of the function $f$ which guranteed $\alpha$ to be 0 .

Taliaferro, in his series of papers (see for e.g. [15], [16], [8]) studied the isolated singularities of non-linear elliptic inequalities. In [16] the author studied the asymptotic behaviour of the positive solution of the differential inequality

$$
0 \leq-\Delta u \leq f(u)
$$

\footnotetext{
* Corresponding author

Email addresses: dhanya.tr@gmail.com (Dhanya Rajendran), abhishek@math.tifrbng.res.in (Abhishek Sarkar )
} 
in a punctured domain under various assumptions on $f$. If $N \geq 3$ and the function $f$ has a "supercritical" growth as in Lions[10], (i.e. $\lim _{t \rightarrow \infty} \frac{f(t)}{t^{N-2}}=\infty$,) then there exists arbitrarily 'large solutions' of (1.1). When $N=2$, it was proved that there exists a punctured neighborhood of the origin such that (1.1) admits arbitrarily large solutions near the origin, provided that $\log f(t)$ has a superlinear growth at infinity. Moreover author characterizes the singularity at the origin of all solutions $u$ of (1.1) when $\log f(t)$ has a sublinear growth. Later Taliaferro, Ghergu and Moradifam in [8] generalized these results to polyharmonic inequalities.

The study of the polyharmonic equations of the type $(-\Delta)^{m} u=h(x, u)$ is associated to splitting the equation into a non-linear coupled system involving Laplace operator alone. Orsina and Ponce[12] proved the existence of solutions to

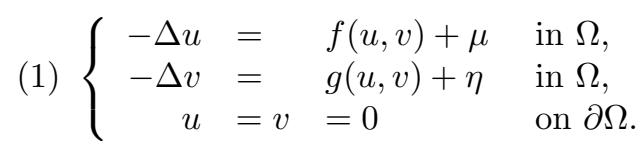

with the assumption that the continuous functions $f$ and $g$ are non increasing in first and second variables respectively with $f(0, t)=g(s, 0)=0$. But here the authors assumed that $\mu$ and $\eta$ are diffusive measures and Dirac distribution is not a diffusive measure. Considerable amount of existence/non-existence results have been proved for the problem (1) when $f$ is a function of $v$ alone and $g$ depends only on $u$ and $\mu, \eta$ are Radon measures. For eg. see [1] where the authors assumed $f(u, v)=v^{p}, g(u, v)=u^{q}$ and with non-homogenous boundary condition. In [6] authors dealt with sign changing functions $f$ and $g$, with a polynomial type growth at infinity and the measure $\mu$ and $\eta$ were assumed to be multiples of $\delta_{0}$.

Our paper is closely related to the work of Soranzo [14] where author considers the equation:

$$
\Delta^{2} u=|x|^{\sigma} u^{p} \text { with } u>0,-\Delta u>0 \text { in } \Omega \subset \mathbb{R}^{N}, N \geq 4 \text { and } \sigma \in(-4,0) .
$$

A complete description of the singularity was provided when $1<p<\frac{N+\sigma}{N-4}$ for $N \geq 5$, or $1<p<\infty$ when $N=4$. In this work we prove that the results of Soranzo can be improved for the dimension $N=4$ by replacing $u^{p}$ by more general exponential type function.

\section{Preliminaries}

We assume that $\Omega$ is a bounded open set in $\mathbb{R}^{N}, N \geq 4$ with smooth boundary and $0 \in \Omega$. We denote $\Omega^{\prime}$ to be $\Omega \backslash\{0\}$. In this section we discuss some of the well known results for biharmonic operator.

Theorem 2.1. (Brezis - Lions [2]) Let $u \in L_{\text {loc }}^{1}\left(\Omega^{\prime}\right)$ be such that $\Delta u \in L_{\text {loc }}^{1}\left(\Omega^{\prime}\right)$ in the sense of distributions in $\Omega^{\prime}, u \geq 0$ in $\Omega$ such that

$$
-\Delta u+a u \geq g \text { a.e in } \Omega,
$$

where $a$ is a positive constant and $g \in L_{\text {loc }}^{1}(\Omega)$. Then there exist $\varphi \in L_{\text {loc }}^{1}(\Omega)$ and $\alpha \geq 0$ such that

$$
-\Delta u=\varphi+\alpha \delta_{0} \text { in } \mathcal{D}^{\prime}\left(B_{R}\right)
$$

where $\delta_{0}$ is the Dirac mass at origin.In particular, $u \in M_{l o c}^{p}\left(B_{R}\right)^{1}$ where $p=N / N-2$ when $N \geq 3$ and $1 \leq p<\infty$ is arbitrary when $N=2$.

Theorem 2.2. (Weyl Lemma, Simader[13]) Suppose $G \subset \mathbb{R}^{N}$ be open and let $u \in L_{\text {loc }}^{1}(G)$ satisfies

$$
\int_{G} u \Delta^{2} \varphi d x=0 \text { for all } \varphi \in C_{c}^{\infty}(G) \text {, i.e. } \Delta^{2} u=0 \text { in } \mathcal{D}^{\prime}(G) \text {. }
$$

Then there exists $\tilde{u} \in C^{\infty}(G)$ with $\Delta^{2} \tilde{u}=0$ and $u=\tilde{u}$ a.e in $G$.

\footnotetext{
${ }^{1} M_{l o c}^{p}\left(B_{R}\right)$ denotes the Marcinkeiwicz space
} 
Theorem 2.3. (Weak maximum principle:) Let $u \in W^{4, r}(\Omega)$ be a solution of

$$
\begin{cases}\Delta^{2} u=f(x) \geq 0 & \text { in } \Omega \\ u \geq 0,-\Delta u \geq 0 & \text { on } \partial \Omega\end{cases}
$$

Then we have $u \geq 0$ and $-\Delta u \geq 0$ in $\Omega$.

Proof of maximum principle easily follows by splitting the equation into a (coupled) system of second order PDE's say: $w=-\Delta u$ and $-\Delta w=f$ with the corresponding boundary conditions. Using similar ideas we can infact prove a maximum principle with weaker assumptions on the the smoothness of $u$, which is stated below:

Theorem 2.4. Let $u, \Delta u \in L^{1}(\Omega)$ and $\Delta^{2} u \geq 0$ in the sense of distributions. Also assume that $u, \Delta u$ are continuous near $\partial \Omega$ and $u>0,-\Delta u>0$ near $\partial \Omega$. Then $u(x) \geq 0$ in $\Omega$.

Definition 2.1. Fundamental solution of $\Delta^{2}$ is defined as a locally integrable function $\Phi$ in $\mathbb{R}^{N}$ for which $\Delta^{2} \Phi=\delta_{0}$ and precisely expressed as

$$
\Phi(x)=a_{N}\left\{\begin{array}{lll}
|x|^{4-N} & \text { if } & N \geq 5 \\
\log \frac{5}{|x|} & \text { if } & N=4 \\
|x| & \text { if } & N=3 \\
|x|^{2} \log \frac{5}{|x|} & \text { if } & N=2
\end{array}\right.
$$

for some constant $a_{N}>0$.

Theorem 2.5. Suppose $g: \Omega^{\prime} \times[0, \infty) \rightarrow \mathbb{R}^{+}$be a measurable function and let $u, \Delta u$ and $\Delta^{2} u \in$ $L_{l o c}^{1}\left(\Omega^{\prime}\right)$. Let $\Delta^{2} u=g(x, u)$ in $\mathcal{D}^{\prime}\left(\Omega^{\prime}\right)$ with $u \geq 0$ and $-\Delta u \geq 0$ a.e in $\Omega^{\prime}$. Then $u, g(x, u) \in L_{l o c}^{1}(\Omega)$ and there exist a non-negative constants $\alpha, \beta$ such that $\Delta^{2} u=g(x, u)+\alpha \delta_{0}-\beta \Delta \delta_{0}$ in $\mathcal{D}^{\prime}(\Omega)$.

Proof: Let us write $w=-\Delta u$. Then $-\Delta w=g(x, u) \geq 0$ in $\mathcal{D}^{\prime}\left(\Omega^{\prime}\right)$ and also given that $w, g(x, u) \in$ $L_{l o c}^{1}\left(\Omega^{\prime}\right)$. Now as a direct application of Brezis-Lions Theorem 4.4, we obtain

$$
-\Delta w=g(x, u)+\alpha \delta_{0} \text { for some } \alpha \geq 0
$$

and $w, g(x, u) \in L_{l o c}^{1}(\Omega)$. Since $-\Delta u=w \geq 0$ in $\Omega^{\prime}$ again by Theorem $4.4 u \in L_{l o c}^{1}(\Omega)$ and

$$
-\Delta u=w+\beta \delta_{0} \text { for some } \beta \geq 0 .
$$

Now substituting $w=-\Delta u-\beta \delta_{0}$ in $(2.2)$ we get

$$
\Delta^{2} u=g(x, u)+\alpha \delta_{0}-\beta \Delta \delta_{0} .
$$

Extending $g(x, u)$ to be zero outside $\Omega$ we get $\Delta^{2}(u-f(u) * \Phi-\alpha \Phi-\beta \Gamma)=0$ in $\mathcal{D}^{\prime}(\Omega)$. By Weyl's lemma for biharmonic operators, there exists a biharmonic function $h \in C^{\infty}(\Omega)$ and

$$
u=g(x, u) * \Phi+\alpha \Phi+\beta \Gamma+\text { h a.e in } \Omega .
$$

Note that $\Gamma(x)$ belongs to Marcinkeiwicz space $M^{\frac{N}{N-2}}(\Omega)$ when $N \geq 2$. By the property of the convolution of an $L^{1}$ function with the functions in $M^{\frac{N}{N-2}}\left(\mathbb{R}^{N}\right)$ we obtain $u \in M_{l o c}^{\frac{N}{N-2}}(\Omega)$.

The above result has been proved in [14](see Theorem 2) as an application of their main result on the system of equations. Proof is essentially based on the idea of Brezis-Lions type estimates. We have instead given a direct alternative proof for the same result. Theorem 2.5 can be extended for polyharmonic operator in a standard way, for details see Theorem 4.1 . 


\section{Biharmonic operator in $\mathbb{R}^{4}$}

In this section we will restrict ourselves to the dimension $N=4$ and $g(x, u)$ to take a specific form $g(x, u)=a(x) f(u)$. Let $\Omega$ be a bounded open set in $\mathbb{R}^{4}, 0 \in \Omega$ and denote $\Omega^{\prime}=\Omega \backslash\{0\}$. We assume

(H1) $f:[0, \infty) \longrightarrow[0, \infty)$ is a continuous function which is nondecreasing in $\mathbb{R}^{+}$and $f(0)=0$.

(H2) $a(x)$ is a non-negative measurable function in $L^{k}(\Omega)$ for some $k>\frac{4}{3}$.

(H3) There exists $r_{0}>0$ such that $\operatorname{essinf}_{B_{r_{0}}} a(x)>0$.

Let $u$ be a measurable function which solves the following problem:

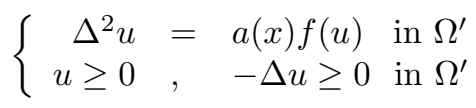

From Theorem 2.5 we know that $u$ is a distributional solution of $\left(P_{\alpha, \beta}\right)$

$$
\left(P_{\alpha, \beta}\right) \quad\left\{\begin{array}{c}
\Delta^{2} u \quad=a(x) f(u)+\alpha \delta_{0}-\beta \Delta \delta_{0} \\
u \geq 0-\Delta u \geq 0 \\
\alpha, \beta \geq 0, u \text { and } a(x) f(u) \in L^{1}(\Omega) .
\end{array}\right\} \text { in } \Omega
$$

The assumption (H3) suggests that the presence of such a weight function does not reduce the singularity of $a(x) f(u)$ at origin. In particular, if $a(x)=|x|^{\sigma}$ for $\sigma \in(-3,0)$, then $a(x)$ satisfies $(H 2)$ and $(H 3)$.

Now assume that

$$
\lim _{t \rightarrow \infty} \frac{f(t)}{t^{2}}=c \in(0, \infty] .
$$

i.e. $f(t)$ grows atleast at a rate of $t^{2}$ near infinity. Then for some $t_{0}$ large enough, we have $f(t) \geq \frac{c}{2} t^{2}$ for all $t \geq t_{0}$. Suppose $u$ is a solution of $\left(P_{\alpha, \beta}\right)$ and $f$ satisfies 3.1. Then we know that for some biharmonic function $h$

$$
u(x)=a(x) f(u) * \Phi+\alpha \Phi+\beta \Gamma+h \text { a.e in } \Omega
$$

where $\Phi$ is the fundamental solution of biharmonic operator in $\mathbb{R}^{4}$ and $\Gamma$ is the fundamental solution of $-\Delta$ in $\mathbb{R}^{4}$. Since $\alpha$ and $a(x) f(u)$ are non-negative, we have $u(x) \geq \beta \Gamma(x)+h(x)$. If $\beta \neq 0$, fix an $\tilde{r} \in\left(0, r_{0}\right)$ such that $u(x) \geq \frac{\beta}{2 \pi^{2}|x|^{2}} \geq t_{0}$ whenever $|x|<\tilde{r}$. Now,

$$
\int_{B_{\tilde{r}}} a(x) f(u) \geq C \int_{B_{\tilde{r}}}|x|^{-4}=\infty
$$

which is a contradiction since $a(x) f(u) \in L^{1}(\Omega)$. Thus $\beta=0$ if $f(t)$ grows at a rate faster than $t^{2}$ near infinity. We state this result in the next lemma.

Lemma 3.1. Let $f$ satisfies the condition (3.1) and $u$ solves $(P)$. Then for some $\alpha$ non-negative $\Delta^{2} u=a(x) f(u)+\alpha \delta_{0}$ in $\mathcal{D}^{\prime}(\Omega)$.

Now onwards we assume that $f$ satisfies (3.1). We would like to address following questions in this paper:

1. Can we find a sharp condition on $f$ that determines whether $\alpha=0$ or not in $\left(P_{\alpha, 0}\right)$ ?

2. If $\alpha=0$, is it true that $u$ is regular in $\Omega$ ? 
Definition 3.1. We call $f$ a sub-exponential type function if

$$
\lim _{t \rightarrow \infty} f(t) e^{-\gamma t} \leq C \quad \text { for } \quad \text { some } \quad \gamma, \mathrm{C}>0 .
$$

We call $f$ to be of super-exponential type if it is not a sub-exponential type function.

We will show that the above two questions can be answered based on the non-linearity being a sub-exponential type function or not.

Theorem 3.1. (Removable Singularity) Let $f$ be a super-exponential type function and $u$ is a distributional solution of $(P)$. Then $u$ extends as a distributional solution of $\left(P_{0,0}\right)$.

Proof: Given $u$ solves $(P)$, we know that $\Delta^{2} u=a(x) f(u)+\alpha \delta_{0}-\beta \Delta \delta_{0}$ for some $\alpha, \beta \geq 0$. To show the extendability of the distributional solution we need to prove $\alpha=\beta=0$. Since $f$ is of super exponential type function, from Lemma 3.1 it is clear that $\beta=0$. Let us assume that $\alpha>0$ and derive a contradiction. Note that we can find an $r$ small enough such that $u(x) \geq-\frac{\alpha}{16 \pi^{2}} \log |x|$ whenever $|x|<r$. Since $f$ is not a sub-exponential type function, for a given $\gamma>0$ there exists $t_{0}>0$ such that $f(t) \geq e^{\gamma t}$ for all $t \geq t_{0}$. Thus,

$$
f(u(x)) \geq f\left(-\frac{\alpha}{16 \pi^{2}} \log |x|\right) \geq e^{-\frac{\gamma \alpha}{16 \pi^{2}} \log |x|}, \text { for }|x|<<1 .
$$

Now if we choose $\gamma=\frac{64 \pi^{2}}{\alpha}$ in the above inequality, it contradicts the fact $a(x) f(u) \in L^{1}(\Omega)$. Thus $\alpha=\beta=0$ in $\left(P_{\alpha, \beta}\right)$.

Theorem 3.2. If $f(t)=t^{p}$ where $1 \leq p<\frac{4+\sigma}{2}$ and $a(x)=|x|^{\sigma}$, for $\sigma \in(-2,0)$ then $\left(P_{\alpha, \beta}\right)$ is solvable for $\alpha, \beta$ small enough.

Proof follows from Theorem 4(ii)) of Soranzo[14]. The idea was to split the equation into a coupled system and find a sub and super solution for the system. In the next theorem when $f$ satisfies 3.1, we find a super solution for $\left(P_{\alpha, 0}\right)$ directly without splitting the equation into a coupled system and then use the idea of monotone iteration to show the existence of a non-negative solution for $\alpha$ small enough. When $\beta \neq 0$, such a direct monotone iteration technique is not applicable as $\Delta \delta_{0}$ is not a positive or a negative distribution, ie $\phi \geq 0$, does not imply $\left\langle\Delta \delta_{0}, \phi\right\rangle \geq 0$ or $\leq 0$.

Theorem 3.3. Let $f$ and a satisfy the hypotheses $(H 1)-(H 3)$. Additionally assume $\lim _{t \rightarrow \infty} \frac{f(t)}{t^{2}}=$ $c \in(0, \infty]$. Then there exists an $\alpha_{*}>0$ such that for all $\alpha \leq \alpha_{*}$ the problem $\left(P_{\alpha, 0}\right)$ admits a solution in $B_{r}(0)$.

Proof: We use the idea of sub and super solution to construct a distributional solution for $\left(P_{\alpha, 0}\right)$ for $\alpha$ small enough. Clearly $u_{0}=0$ is a subsolution for $\left(P_{\alpha, 0}\right)$. Given that $f$ is a sub-exponential type nonlinearity, there exists a $\gamma>0$ and a $C>0$, such that $f(t) \leq C e^{\gamma t}$ for all $t \in \mathbb{R}^{+}$.

Now define

$$
\bar{u}(x)=\frac{-\log |x|+C \phi}{\gamma} \text { in } B_{1}(0)
$$

where $\phi$ is the unique solution of the following Navier boundary value problem,

$$
\left\{\begin{array}{c}
\Delta^{2} \phi=-\frac{a(x)}{|x|} \log |x| \text { in } B_{1}(0) \\
\phi=0=\Delta \phi \text { on } \partial B_{1}(0) .
\end{array}\right.
$$

We notice that since $a(x) \in L^{k}(\Omega)$, for some $k>\frac{4}{3}$, the term $a(x)|x|^{-1} \log |x| \in L^{p}\left(B_{1}\right)$ for some $p>1$. Hence the existence of a unique weak solution $\phi \in W^{4, p}\left(B_{1}\right)$ is guarenteed by Gazzolla [7], Theorem 2.20. Now by maximum principle we have $\phi \geq 0,-\Delta \phi \geq 0$.

Therefore,

$$
\bar{u} \geq 0 \text { in } B_{1}(0),
$$




$$
-\Delta \bar{u}=\frac{2}{\gamma|x|^{2}}-\frac{C}{\gamma} \Delta \phi \geq 0
$$

and

$$
\Delta^{2} \bar{u}=\frac{\delta_{0}}{8 \pi^{2} \gamma}+\frac{C}{\gamma|x|} a(x)|\log | x|| .
$$

Note that $a(x) f(\bar{u}) \leq \frac{C}{|x|} a(x) e^{C \phi}$. By Sobolev embedding, we know $W^{4, p}(\Omega) \hookrightarrow C(\bar{\Omega})$, and hence $e^{C \phi}$ is bounded in $B_{1}(0)$. Now we fix an $r>0$ where $e^{C \phi} \leq \frac{|\log | x||}{\gamma}$ in $B_{r}(0)$. We let $\Omega=B_{r}(0)$ (where $r$ depends only on $\gamma$ and $C$ ) be a strict subdomian of $B_{1}(0)$ where $\frac{C}{\gamma|x|} a(x)|\log | x|| \geq$ $a(x) f(\bar{u})$. Now from the choice of $r$ and equations 3.4), (3.5) and (3.6) it is obvious that $\bar{u}$ is a super solution of $\left(P_{\alpha, 0}\right)$ where $\alpha=\frac{1}{8 \pi^{2} \gamma}$. Now let us define inductively with $u_{0}=0$

$$
\left(P_{\alpha, 0}^{n}\right)\left\{\begin{aligned}
\Delta^{2} u_{n}=a(x) f\left(u_{n-1}\right)+\alpha \delta_{0} & \text { in } \mathcal{D}^{\prime}(\Omega) \\
u_{n}>0,-\Delta u_{n}>0 & \text { in } \Omega \\
u_{n}=\Delta u_{n}=0 & \text { on } \partial \Omega
\end{aligned}\right.
$$

Existence of such a sequence $\left\{u_{n}\right\}$ can be obtained by writing $u_{n}=w_{n}+\alpha \Phi$ where

$$
\left\{\begin{array}{l}
\Delta^{2} w_{n}=a(x) f\left(u_{n-1}\right) \text { in } \Omega \\
w_{n}=-\alpha \Phi, \Delta w_{n}=-\alpha \Delta \Phi \text { on } \partial \Omega, \\
w_{n} \in W^{4, r}(\Omega) \text { for some } r>1 .
\end{array}\right.
$$

Existence of $w_{1}$ is clear since $f(0)=0$ and from Theorem 2.2 of [7]. First let us show the positivity of $u_{1}$ and $-\Delta u_{1}$ in $\Omega$. Since $w_{1}$ is bounded, we can choose $\epsilon$ small enough so that $u_{1}=w_{1}+\alpha \Phi>0$ and $-\Delta u_{1}>0$ in $B_{\epsilon}$. In $\Omega \backslash B_{\epsilon}$ by weak comparison principle we can show that $u_{1}>0$ and $-\Delta u_{1}>0$. Next we need to show that $u_{1} \leq \bar{u}$. Note that by construction, $\bar{u}>0$ and $-\Delta \bar{u}>0$ in $\overline{B_{r}} \backslash\{0\}$. Then, $\bar{u}-u_{1}$ satisfies the set of equations

$$
\left\{\begin{aligned}
\Delta^{2}\left(\bar{u}-u_{1}\right) \geq 0 & \text { in } \mathcal{D}(\Omega) \\
\bar{u}-u_{1}>0,-\Delta\left(\bar{u}-u_{1}\right)>0 & \text { near } \partial \Omega .
\end{aligned}\right.
$$

Now using the maximum principle for distributional solutions (Theorem 2.4) we find $u_{1} \leq \bar{u}$.

Assume that there exists a function $u_{k}$ solving $\left(P_{\alpha, 0}^{k}\right)$ for $k=1,2 \cdots n$ and

$$
0 \leq u_{1} \leq u_{2} \ldots \leq u_{n} \leq \bar{u} \text { in } \Omega \text {. }
$$

Since $f$ is non-decreasing we have $a(x) f\left(u_{n}\right) \in L^{p}(\Omega)$, for some $p>1$. Thus by Sobolev embedding there exists a $w_{n+1} \in C(\bar{\Omega}) \cap W^{4, p}(\Omega)$. Also,

$$
\left\{\begin{array}{lc}
\Delta^{2}\left(u_{n+1}-u_{n}\right)=a(x) f\left(u_{n}\right)-a(x) f\left(u_{n-1}\right) \geq 0 & \text { in } \Omega \\
u_{n+1}=u_{n}, \Delta u_{n+1}=\Delta u_{n} & \text { on } \partial \Omega .
\end{array}\right.
$$

Again from weak comparison principle $0<u_{n} \leq u_{n+1}$ and $0 \leq-\Delta u_{n} \leq-\Delta u_{n+1}$. As before one can show that $u_{n+1} \leq \bar{u}$. Now if we define $u(x)=\lim _{n \rightarrow \infty} u_{n}(x)$ one can easily verify that $u$ is a solution of $\left(P_{\alpha, 0}\right)$ for $\alpha=\frac{1}{8 \pi^{2} \gamma}$. For a given $f$ sub-exponential type function we have found a ball of radius $r$ such that $\left(P_{\alpha, 0}\right)$ posed on $B_{r}(0)$ has a solution $u_{\alpha}$ for $\alpha=\frac{1}{8 \pi^{2} \gamma}$. This solution $u_{\alpha}$ is a supersolution for $\left(P_{\alpha^{\prime}, 0}\right)$ posed in $B_{r}(0)$ and for $\alpha^{\prime} \in(0, \alpha)$. Thus one can repeat the previous iteration and show that for all $\alpha^{\prime} \in(0, \alpha)$ there exists a weak solution for $\left(P_{\alpha^{\prime}, 0}\right)$ in $B_{r}(0)$.

Corollary 3.1. Suppose for a given $\gamma>0$ there exists a $C_{\gamma}$ such that $f(t) \leq C_{\gamma} e^{\gamma t}$ for all $t \in \mathbb{R}^{+}$. Then $\left(P_{\alpha, 0}\right)$ has a solution in $B_{r_{\alpha}}(0)$ for all $\alpha \in(0, \infty)$. In particular if $f(t)=t^{p}, p>2$ or $e^{t^{\delta}}, \delta<1$ then $\left(P_{\alpha, 0}\right)$ is solvable for all $\alpha>0$. 
Next we recall a Brezis-Merle [3] type of estimate for Biharmonic operator in $\mathbb{R}^{4}$. Let $h$ be a distributional solution of

$$
\text { (2) } \begin{cases}\Delta^{2} h=f & \text { in } \Omega \\ h=\Delta h=0 & \text { on } \partial \Omega\end{cases}
$$

where $\Omega$ is a bounded domain in $\mathbb{R}^{4}$.

Theorem 3.4. (C.S Lin [9]) Let $f \in L^{1}(\Omega)$ and $h$ is a distributional solution of (2). For a given $\delta \in\left(0,32 \pi^{2}\right)$ there exists a constant $C_{\delta}>0$ such that the following inequality holds:

$$
\int_{\Omega} \exp \left(\frac{\delta h}{\|f\|_{1}}\right) d x \leq C_{\delta}(\operatorname{diam} \Omega)^{4}
$$

where diam $\Omega$ denote the diameter of $\Omega$.

Theorem 3.5. Let $f$ be a sub-exponential type function. Let $u$ be a solution of $\left(P_{0,0}\right)$ with $u=\Delta u=0$ on $\partial \Omega$. Then $u$ is regular in $\Omega$.

Proof: Let $u$ be a solution of $\Delta^{2} u=a(x) f(u)$ in $\Omega$ with Navier boundary conditions. Write $g(x)=a(x) f(u)$, then $g \in L^{1}(\Omega)$. Fix a $l>0$ and split $g=g_{1}+g_{2}$ where $\left\|g_{1}\right\|_{1}<\frac{1}{l}$ and $g_{2} \in L^{\infty}(\Omega)$. Let $u_{2}$ be the unique solution of

$$
\left\{\begin{array}{l}
\Delta^{2} u_{2}=g_{2} \text { in } \Omega \\
u_{2}=0, \Delta u_{2}=0 \text { on } \partial \Omega .
\end{array}\right.
$$

Then

$$
\left\{\begin{array}{l}
\Delta^{2} u_{1}=g_{1} \text { in } \Omega \\
u_{1}=0, \Delta u_{1}=0 \text { on } \partial \Omega .
\end{array}\right.
$$

Choosing $\delta=1$ in Theorem 3.4, we find $\int_{\Omega} \exp \left(\frac{\left|u_{1}\right|}{\left\|g_{1}\right\|_{1}}\right)<C_{1}(\operatorname{diam} \Omega)^{4}$. Thus $e^{l\left|u_{1}\right|} \in L^{1}(\Omega)$. Since $u_{2} \in L^{\infty}(\Omega)$, we have $e^{l|u|} \in L^{1}(\Omega)$ for all $l>0$. We use this higher intergrability property of $u$ in establishing its regularity.

We can show that $a(x) f(u) \in L^{r}(\Omega)$ for some $r>1$. In fact,

$$
\begin{aligned}
\int_{\Omega}(a(x) f(u))^{r} & \leq \tilde{C} \int_{\Omega} a(x)^{r} e^{\gamma r u} \\
& \leq C_{2}\left(\int_{\Omega} a(x)^{p r}\right)^{1 / p}\left(\int_{\Omega} e^{p^{\prime} \gamma r u}\right)^{1 / p^{\prime}}<\infty
\end{aligned}
$$

if we choose $p, r>1$ close enough to 1 so that $1<p . r \leq k$, where $a(x) \in L^{k}(\Omega)$. Now let $v$ be the unique weak solution of

$$
\left\{\begin{array}{l}
\Delta^{2} v=a(x) f(u) \text { in } \Omega \\
v=0, \Delta v=0 \text { on } \partial \Omega
\end{array}\right.
$$

We have $v \in C^{3, \gamma^{\prime}}(\bar{\Omega})$ for all $\gamma^{\prime} \in(0,1)$. Now $u=v+h$ for some biharmonic function $h$. Therefore $u \in C^{3, \gamma^{\prime}}(\Omega)$.

Remark 3.1. The previous theorem is true even if $a(x) \in L^{k}(\Omega)$ for some $k>1$.

When $f$ is super exponential in nature an arbitrary solution of $\Delta^{2} u=a(x) f(u)$ in $\mathcal{D}^{\prime}(\Omega)$ need not be bounded. We consider the following example.

Example 3.1. Let $w(x)=(-4 \log |x|)^{\frac{1}{\mu}}$ for some $\mu>1$. Then one can verifty that whenever $x \neq 0$,

$$
\Delta^{2} w=b_{1} e^{w^{\mu}} w^{1-4 \mu}\left[b_{2} w^{2 \mu}-b_{3}\right]
$$

for some positive constants $b_{i}$. Since $f(w)=b_{1} e^{w^{\mu}} w^{1-4 \mu}\left[b_{2} w^{2 \mu}-b_{3}\right]$ is super exponential in nature, $w$ extends as an unbounded distributional solution of $\Delta^{2} w=f(w)$ in $B_{r}(0)$ for $r$ small enough. 


\section{Polyharmonic Operator in $\mathbb{R}^{2 m}$}

We suppose $\Omega$ is a bounded domain in $\mathbb{R}^{N}, N \geq 2 m$ with smooth boundary and $0 \in \Omega$. We denote $\Omega^{\prime}$ as $\Omega \backslash\{0\}$.

Theorem 4.1. Suppose $g: \Omega^{\prime} \times[0, \infty) \rightarrow \mathbb{R}^{+}$is a measurable function and $\Delta^{k} u \in L_{\text {loc }}^{1}\left(\Omega^{\prime}\right)$ for $k=0,1, . . m$. Let $(-\Delta)^{m} u=g(x, u)$ in $\mathcal{D}^{\prime}\left(\Omega^{\prime}\right)$ with $(-\Delta)^{k} u \geq 0$ for $k=0,1, . ., m-1$ a.e in $\Omega^{\prime}$. Then $u, g(x, u) \in L_{\text {loc }}^{1}(\Omega)$ and there exist non-negative constants $\alpha_{0}, \ldots, \alpha_{m-1}$ such that $(-\Delta)^{m} u=g(x, u)+\sum_{i=0}^{m-1} \alpha_{i}(-\Delta)^{i} \delta_{0}$ in $\mathcal{D}^{\prime}(\Omega)$.

Now we restrict ourselves to dimension $N=2 m$ and $g(x, u)$ to take a specific form $g(x, u)=$ $a(x) f(u)$. Throughout this section we make the following assumption:

$\left(H 1^{\prime}\right) f:[0, \infty) \mapsto[0, \infty)$ is a continuous function which is non-decreasing in $\mathbb{R}^{+}$and $f(0)=0$.

$\left(H 2^{\prime}\right) a(x)$ is non negative measurable function in $L^{k}(\Omega)$ for some $k>\frac{2 m}{2 m-1}$.

$\left(H 3^{\prime}\right)$ There exists $r_{0}>0$ such that $\operatorname{essinf}_{B_{r_{0}}} a(x)>0$.

Let $u$ be a measurable function which satisfies the problem below,

$$
\left(P^{1}\right) \quad\left\{\begin{array}{rlrl}
(-\Delta)^{m} u & =a(x) f(u) & \text { in } \Omega^{\prime} \\
(-\Delta)^{k} u \geq 0 \text { in } \Omega^{\prime}, & k=0, . ., m-1 \\
u & \in C^{2 m}(\bar{\Omega} \backslash\{0\}) .
\end{array}\right.
$$

Then by 4.1 we know that $u$ is a distribution solution of $\left(P_{\alpha_{0}, . ., \alpha_{m-1}}^{1}\right)$

$$
\left(P_{\alpha_{0}, . ., \alpha_{m-1}}^{1}\right) \quad\left\{\begin{array}{c}
(-\Delta)^{m} u=a(x) f(u)+\sum_{i=0}^{m-1} \alpha_{i}(-\Delta)^{i} \delta_{0} \text { in } \Omega \\
(-\Delta)^{k} u \geq 0, \quad k=0, . ., m-1 \text { in } \Omega^{\prime} \\
\alpha_{i} \geq 0, \text { for } i=0, . ., m-1 \text { and } u, a(x) f(u) \in L^{1}(\Omega) .
\end{array}\right.
$$

In [4], Soranzo et.al considered a specific equation $(-\Delta)^{m} u=|x|^{\sigma} u^{p}$ in $\Omega^{\prime}$, with $\sigma \in(-2 m, 0)$ and $(-\Delta)^{k} u \geq 0$, for $k=0,1, \ldots m$. By Corollary 1 of [4], if $N=2 m$ and $p>\max \left\{1, \frac{N+\sigma}{2}\right\}$ then $\alpha_{1}=\alpha_{2}=\cdots=\alpha_{m-1}=0$ in $\left(P_{\alpha_{0}, \ldots \alpha_{m-1}}^{1}\right)$. This result can be sharpened for any weight function $a(x)$ satisfying $(H 3)$ in a standard way and we skip the details of the proof.

Remark 4.1. Let $u$ satisfy $\left(P^{1}\right)$ and $\lim _{t \rightarrow \infty} \frac{f(t)}{t^{m}}=c \in(0, \infty]$. Then we have $\alpha_{1}=\alpha_{2}=. .=$ $\alpha_{m-1}=0$ in $\left(P_{\alpha_{0}, \ldots, \alpha_{m-1}}^{1}\right)$ and hence $u$ is a distributional solution of $(-\Delta)^{m} u=a(x) f(u)+\alpha_{0} \delta_{0}$ in $\Omega$.

Now the following theorem gives us a sharp condition on $f$ which determines $\alpha_{0}=0$ in $\left(P_{\alpha_{0}, 0, \ldots, 0}^{1}\right)$ and the proof is as similar to Theorem 3.1.

Theorem 4.2. Let $f$ be a super-exponential type function and $u$ is distribution solution of $\left(P^{1}\right)$. Then $u$ extends as a distributional solution of $\left(P_{0,0, ., 0}^{1}\right)$.

Theorem 4.3. Let $f$ and a satisfy the hypotheses $\left(H 1^{\prime}\right)-\left(H 3^{\prime}\right)$. Additionally assume $\lim _{t \rightarrow \infty} \frac{f(t)}{t^{m}}=$ $c \in(0, \infty]$. Then there exists an $\alpha_{0}>0$ such that for all $\alpha \leq \alpha_{0}$ the problem $\left(P_{\alpha, 0, \ldots 0}^{1}\right)$ admits a solution in $B_{r}(0)$, where the radius of the ball depends on the nonlinearity $f$. 
Proof: We proceed as in Theorem 3.3, by constructing sub and super distributional solution for $\left(P_{\alpha, 0, ., 0}^{1}\right)$ for all $\alpha$ small enough. We note that $u_{0}=0$ is a sub-solution, and let

$$
\bar{u}(x)=\frac{-\log |x|+C \phi}{\gamma} \text { in } B_{1}(0)
$$

where $\phi$ is the unique solution of the following Navier boundary value problem,

$$
\left\{\begin{aligned}
(-\Delta)^{m} \phi & =-\frac{a(x)}{|x|} \log |x| \text { in } B_{1}(0) \\
\phi=\Delta \phi & =. .=(\Delta)^{m-1} \phi \text { on } \partial B_{1}(0)
\end{aligned}\right.
$$

Then $\bar{u}$ is a supersolution of $\left(P_{\alpha, 0 \ldots 0}^{1}\right)$ in a small ball $B_{r}(0)$. Rest of the proof follows exactly as in the case of biharmonic operator.

Next we state a Brezis-Merle type of type of estimates for poly-harmonic operator in $\mathbb{R}^{2 m}$.

Theorem 4.4. (Martinazzi [11]) Let $f \in L^{1}\left(B_{R}\left(x_{0}\right)\right), B_{R}\left(x_{0}\right) \subset \mathbb{R}^{2 m}$, and let $v$ solve

$$
\left\{\begin{array}{c}
(-\Delta)^{m} v=f \text { in } B_{R}\left(x_{0}\right) \\
v=\Delta^{2} v=\ldots .=\Delta^{m-1} v=0 \text { on } \partial B_{R}\left(x_{0}\right)
\end{array}\right.
$$

Then, for any $p \in\left(0, \frac{\gamma_{m}}{\|f\|_{L^{1}\left(B_{R}\left(x_{0}\right)\right)}}\right)$, we have $e^{2 m p|v|} \in L^{1}\left(B_{R}\left(x_{0}\right)\right)$ and

$$
\int_{B_{R}\left(x_{0}\right)} e^{2 m p|v|} d x \leq C(p) R^{2 m}
$$

where $\gamma_{m}=\frac{(2 m-1) !}{2}\left|S^{2 m}\right|$.

Finally with the help of above theorem we prove a regularity result for the polyharmonic operator.

Theorem 4.5. Let $a(x)$ and $f$ satisfies the properties as in $\left(H 1^{\prime}\right)-\left(H 3^{\prime}\right)$ and also assume that $f$ be a sub-exponential type function. Let $u$ be a solution $\left(P_{0,0, \ldots, 0}^{1}\right)$ with $u=\Delta u=\ldots=\Delta^{m-1} u=0$ on $\partial \Omega$. Then $u \in C^{2 m-1, \gamma^{\prime}}(\Omega)$, for all $\gamma^{\prime} \in(0,1)$.

Acknowledgement : Dhanya.R was supported by UGC under Dr.D.S Kothari Postdoctoral fellowship scheme No.F.4-2/2006(BSR)/13-1045. Both the authors would like to thank Prof. S. Prashanth for various useful discussions.

\section{References}

[1] Bidaut-Véron, Marie Françoise; Yarur, Cecilia; Semilinear elliptic equations and systems with measure data: existence and a priori estimates. Adv. Differential Equations 7 (2002), no. 3, 257-296.

[2] H. Brezis and P.L Lions, A Note on Isolated Singularities for Linear Elliptic Equations, Mathematical Analysis and Applications, Part A Advances in Mathematics Supplementary Studies, Vol. 7A,263-266.

[3] H. Brezis and Frank Merle, Uniform Estimates and blow-up behaviour for solutions of $-\Delta u=$ $V(x) e^{u}$ in two dimensions, Communication in Partial Differential Equations, 16,(8 and 9), 1223-1253 (1991).

[4] Caristi, Gabriella; Mitidieri, Enzo; Soranzo, Ramo;n Isolated singularities of polyharmonic equations. Dedicated to Prof. C. Vinti (Italian) (Perugia, 1996). Atti Sem. Mat. Fis. Univ. Modena 46 (1998), suppl., 257-294. 
[5] Dhanya, R.; Giacomoni, J.; Prashanth, S., Isolated singularities for the exponential type semi-linear elliptic equation in $\mathbb{R}^{2}$. Proc. Amer. Math. Soc. 137 (2009), no. 12, 4099-4107.

[6] García-Huidobro, Marta; Yarur, Cecilia; Existence of singular solutions for a Dirichlet problem containing a Dirac mass. Nonlinear Anal. 74 (2011), no. 8, 2831-2843.

[7] F. Gazzola, H.-C. Grunau, G. Sweers, Polyharmonic Boundary Value Problems, 1st edition, Lecture Notes in Math., vol. 1991, Springer, 2010.

[8] Ghergu, Marius; Moradifam, Amir; Taliaferro, Steven D., Isolated singularities of polyharmonic inequalities. J. Funct. Anal. 261 (2011), no. 3, 660-680.

[9] C.S. Lin, A classification of solutions of a conformally invariant fourth order equation in $\mathbb{R}^{n}$. Comment. Math. Helv. 73 (1998), no. 2, 206-231.

[10] P.L. Lions, Isolated Singularities in Semilinear Problems ,Journal of Diffential Equations 38, 441-450(1980).

[11] Luca Martinazzi, Concentration-compactness phenomena in the higher order Liouville's equation, Journal of Functional Analysis 256(2009), 3743-3741.

[12] Orsina, Luigi; Ponce, Augusto C., Semilinear elliptic equations and systems with diffuse measures. J. Evol. Equ. 8 (2008), no. 4, 781-812.

[13] Simader, Christian G., Mean value formulas, Weyl's lemma and Liouville theorems for $\Delta^{2}$ and Stokes' system. Results Math. 22 (1992), no. 3-4, 761-780.

[14] Soranzo, Ramon Isolated singularities of positive solutions of a superlinear biharmonic equation. Potential Anal. 6 (1997), no. 1, 57-85.

[15] Taliaferro, Steven D., On the growth of superharmonic functions near an isolated singularity. I. J. Differential Equations 158 (1999), no. 1, 28-47.

[16] Taliaferro, Steven D., On the growth of superharmonic functions near an isolated singularity. II. Comm. Partial Differential Equations 26 (2001), no. 5-6, 1003-1026. 